\title{
ABSTRACTS FROM CURRENT LITERATURE
}

Polyarthritis in Rats. W. A. Collier (Eijkman Instituut, Batavia). Geneeskundig Tijdschrift voor Nederlandsche-Indie. Afl. 45, Deel 78, 1938.

IN the course of certain researches among Norwegian rats one animal was met with in which both hind feet were swollen. Material from the affected tissues was injected into four white rats, chosen because of the close relationship of the white rat to the Norwegian strain, and the same symptoms due to a polyarthritis were reproduced. Tissue from an affected joint suitably prepared with aseptic precautions was injected into the plantar region of experimental animals. This produced a slight inflammation at the point of injection which quickly passed off. After an incubation period of usually five to seven days, rarely eight days, the leg began to swell near the tibio-tarsal joint, and sometimes also in the vicinity of the metatarsal joints, and this rapidly increased and in many cases spread to the corresponding joints of the other hind leg, the legs swelling to two or three times the normal size; in a few cases, however, the process remained localised and gradually regressed to normal. Some fluid was found in the joints which was culturally sterile unless secondarily infected; rarely suppuration was observed.

In some animals the arthritis spread to the front legs, and rarely to the end joints of the tail. Some of the animals died, but the others made a slow recovery with subsidence of the swelling.

In many cases a little fluid was found in the pericardium, and a thin pleural exudate poor in cells; very seldom there was also a peritoneal exudate, but in none of these fluids were any bacteria discovered microscopically.

The histological findings were inconclusive and not sufficiently extensive, and need to be repeated; radiological examination showed changes in the joints and adjacent bony tissues.

In further investigations 186 rats were injected subcutaneously in the plantar surface, of which 181 became ill; in fifteen others the infected material was rubbed into scarifications on the legs and 
all were infected; eight were injected subcutaneously in the abdominal wall and five were infected; nine were injected intraperitoneally and four became definitely ill. In the latter cases the formation of pericardial and pleural exudates was specially marked, but the local polyarthritic symptoms were as severe as in the others.

TABLE I.

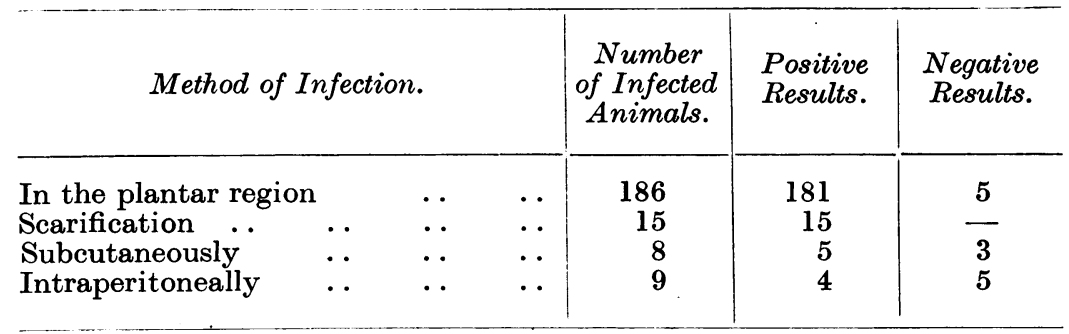

Experiments were carried on to determine if the disease might be communicated by contact, but normal rats placed in the same cage with infected ones were unaffected, nor did they develop immunity; this was also the case when the normal rats were scarified on both hind legs. Observations on the possibility of a prenatal infection were inconclusive.

Experiments were then made with the field rat, which is more distantly related to the Norwegian rat, and it was found to be less susceptible, as shown by a longer incubation period as well as less severe symptoms. Other rodents were less susceptible.

A further series of experiments by W. A. Collier and $\mathbf{H}$. Esseveld were carried out and reported (Geneeskundig Tijdschrift voor Nederlandsch-Indie, Afl. 47, Deel 78, 1938).

The infecting material was obtained by amputating one of the swollen joints, rinsing it with alcohol and then with sterile physiological saline, and finally grinding it in a mortar. The substance obtained was filtered through gauze and injected into healthy animals. The following results were obtained:

Rat $219 c$ was infected in the plantar region on April 2, 1938; nine days later both hind legs showed severe swelling, and the front legs were also affected, though less severely. Cultures were made on ascites agar, Loffler serum, and blood agar from the heart blood, the pleural fluid, liver, testis, brain, and joint fluid 
from the left hind and the front leg. Duplicate cultures were made and half treated aerobically, half anaerobically, and checked after twenty-four, forty-eight and seventy-two hours. All remained sterile with the exception of an aerobic culture from heart blood, which showed a colony of a green streptococcus.

Three other rats were infected with the material from the first; one with blood and brain remained healthy, but the others treated with pleural fluid, liver, testis, and joint fluid all developed polyarthritis. (It is not clear whether the injections were made with mixtures of the substances referred to.-EDITor.) Another rat was injected with the streptococci from the contaminated culture referred to above, but with negative result.

Rat $201 a$ injected in the plantar region with the same result; other rats infected with material from the organs in the same way as in the previous instance; the disease developed in the cases treated from the joints and the blood, but not from the liver or pleural fluid.

Three other rats were infected in the same way and with similar results, and the secondary infections carried out in the same way with some positive and some negative results.

Cultures were made on Löffler plates on account of the work of Fischl, who cultivated corynebacilli from cases of infective arthritis in mice. Levaditi isolated a cocco-bacillus from cases of the same disease, but this also was not found in the present research. The only contaminating organisms met with were "green" streptococci, in one instance from the heart blood and in another from a joint.

These streptococci cannot be held responsible for the arthritis. Out of a total of seventy-nine aerobic and twenty-one anaerobic cultures they were found only in these two instances. In sufferers from many diseases streptococci have been cultivated. Libman pointed out that streptococci are the most important aerobic micro-organisms, acting secondarily, and therefore to be taken into account. The observations of Epstein and Kugel are also quoted; they found streptococci of various strains in a large series: in 40 per cent. from apparently normal heart valves, in 47 per cent. from the heart muscle, in 67 per cent. from the bone marrow, and in 79 per cent. from the blood.

As a further test twenty-four-hour cultures of the green streptococci referred to were injected into the plantar region of rats without any symptoms resulting. 
During previous bacteriological researches on rats other microorganisms were isolated in a few instances-Gram-negative cocci, staphylococci, thick non-motile Gram-positive rods, and a strain of staphylococcus designated B.P., which on inoculation produced localised abscesses which cleared up within a short time. Cultures of these various organisms twenty-four to twenty-eight hours old were injected into the plantar area of white rats, with the following results:

Table II.-Infection Tests with Strains of Bacinli from Rats.

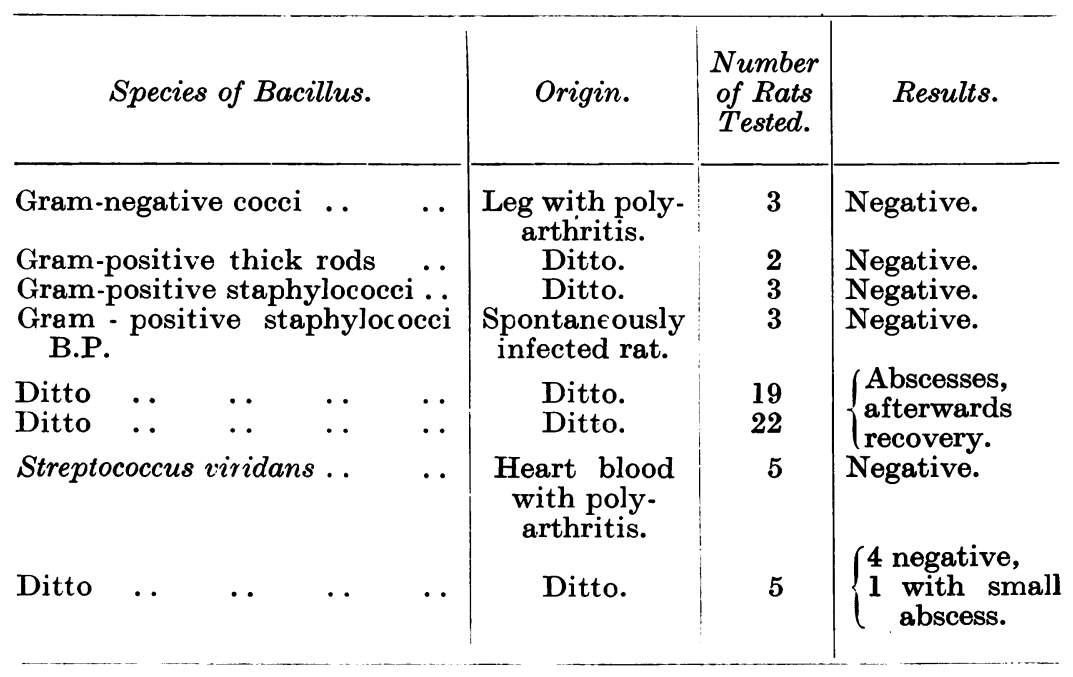

From these tests it is evident that in no case did the infection of healthy rats with these organisms produce symptoms with even the slightest resemblance to polyarthritis. All the rats used in the tests were infected with material from animals with polyarthritis twenty-two to seventy-three days later, and all proved susceptible, so that no immunity had been set up.

It may therefore be presumed that the microbes found in these cultures were either present in the organs concerned or were accidental contaminations. The first is not unlikely in view of the fact that the diseases of many animals are of a very violent nature; in the affected legs as loci minoris resistentiæ secondary infections either from inside or outside may easily occur. The conclusion is therefore drawn that the cause of this rat polyarthritis is either a very difficult or an uncultivatable carrier of bacteriological nature or an ultra-virus. Filtration tests are now 
being carried out. No success has been obtained with microscopical examination even with the dark-field microscope.

The infectious arthritis of mice described by Fischl resembles the polyarthritis of rats in its early stages, but in the later stages there are differences; in mice a generalisation of the disease was never observed, in rat polyarthritis this was always the case. Fischl was always successful in cultivating the infecting organism, the corynebacterium arthritidis muris; in the polyarthritis of rats this was impossible. The corynebacterium is also infectious to rats and guinea-pigs, but mice proved much less susceptible to the microbe of polyarthritis than rats, and guinea-pigs are apparently insusceptible. That the two diseases are distinct thus appears certain.

Each of the organs of twelve infected white rats was used for infection of healthy rats in a further series of experiments, with the following results:

Table III.-Inoculation of White Rats with Substance from INFECTED RATS.

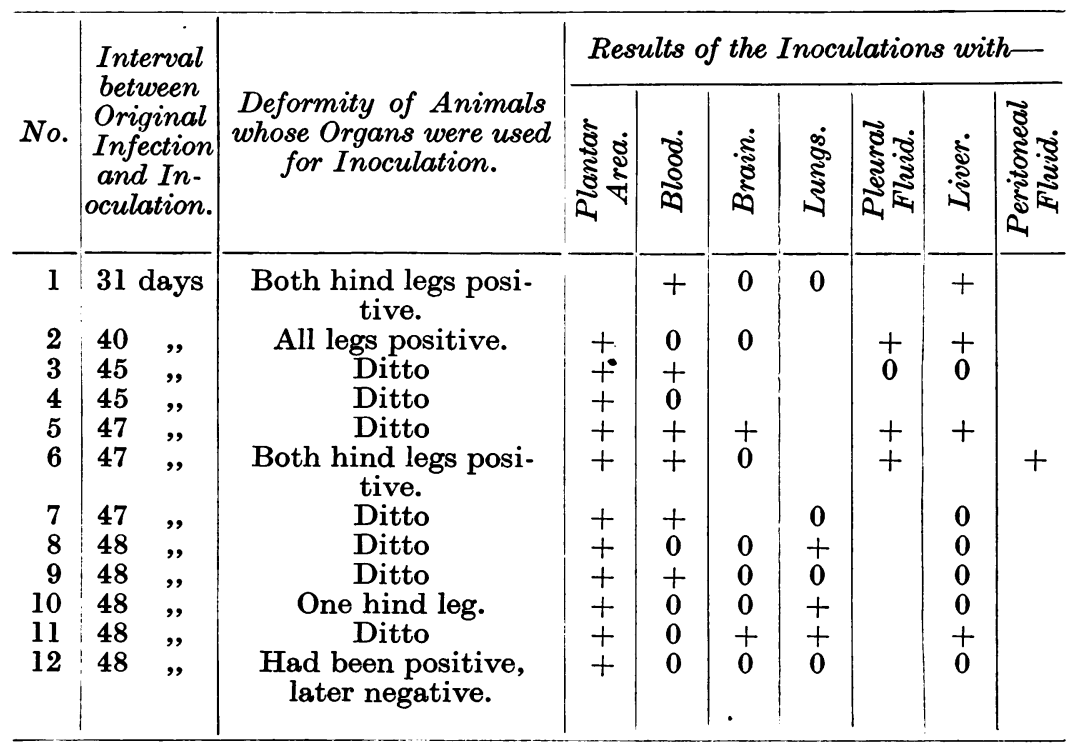

SUMmaRY.-So far no success has been attained in cultivating a micro-organism of ætiological importance from the affected joints in cases of rat polyarthritis. With organs bacteriologically sterile it was repeatedly possible to infect other rats. The 
existence of an infective agent was proved apart from the inflamed joints in the blood, the brain, the lungs, the pleural fluid, the liver, the testis, and the peritoneal fluid.

C. W. B.

REFERENCES

Epstein ANd Kuget (1928): Trans. New York Path. Soc.

Epstein and Kugel (1929): Journ. Inf. Dis., 44, 327.

Fischl, Koech, and Kussat (1931): Zeitsch. f. Hyg., 112, 421.

Levaditi, quoted by Fischi et al., $q . v$.

Lrbman (1923): Journ. Amer. Med. Assoc., 80, 813.

Libman (1928): " New York Acad. Med. Lectures on Medicine and Surgery."

A Serological Test: The B.B.F. Reaction for the Diagnosis of Acute Rheumatism. H. Brokman, J. Brill, and J. Frendzel.

This is an important research directed to establishing a method of value in the diagnosis of acute rheumatism (Bouillaud's disease) by means of a complement-fixation reaction; it is contained in two papers.

1. Compt. Rend. Soc. de Biol., Paris, 1935, vol. cxx., p. 372.The ætiology of the disease being at present unknown, an antigen consisting of an aqueous extract of the organs of an infant dead from the malady was used, because of two possibilities:

(i) In the case of the existence of a specific pathogenic agent it would be found in the extract.

(ii) Rheumatic tissue possesses a certain specificity from the standpoint of morbid anatomy (Aschoff's nodes), and there may be a specificity analogous to that of cancerous or tuberculous tissues.

The technique of the complement-fixation reaction was used. The antigenic extract was prepared in the first instance from the liver and heart of a child dead of acute rheumatisn, and employed in doses of 0.05 and $0 \cdot 1$ c.c. Serum specimens were examined from ten cases of clinically proved acute rheumatism (endocarditis, myocarditis, pericarditis, polyarthritis), a case of chorea, and one of a post-rheumatic affection.

The reaction was positive seven times, suspect once, and negative twice. Chorea gave a negative result. In one case of endo-myopericarditis the test was repeated three times at intervals of fifteen days, and was strongly positive each time; the conclusion is drawn that the affection was active throughout. In a case of chronic rheumatic endocarditis the serum at the first examination gave a negative result. It was only after an exacer- 
bation that the examination of the serum repeated after four weeks gave a sharply positive reaction. In another case the reaction was at first positive; fifteen days later it was only feeble, the inflammatory symptoms, pericarditis and pleurisy having subsided.

To prove the specificity of the reaction two of the positive sera were submitted to the Bordet-Wassermann antigen, with negative result.

Sera from twenty-four patients suffering from diseases other than rheumatism gave a negative result in every case. Pleural fluid from a case of rheumatic pleurisy gave a positive result, while that from a tuberculous pleurisy reacted negatively.

The authors conclude that the complement-fixation reaction with their antigen may be of great practical value in differentiating the malady of Bouillaud from other rheumatic affections, and can serve as an index of the activity of the disease.

2. Klin. Wochenschrift, April, 1937, vol. xvi., p. 502.-In this further report by the same authors an account of more work on the antigen is given, comparison being made of extracts from the liver, spleen, and heart of three children dead of acute rheumatism, with the result that the liver extract was found to give a better result than other organs even when the heart showed Aschoff nodes. This is against possibility no. ii above.

The making of the liver antigen has now been standardised, and the procedure is described as follows: 200 grammes of liver of a child dead of acute rheumatism is minced and put into a flask with 800 c.c. of saline to which 0.5 per cent. of phenol is added as a preservative. The mixture, after shaking for four hours in an apparatus, is left for six weeks in a refrigerator at $4^{\circ} \mathrm{C}$. The fluid is then decanted and filtered through paper; the filtrate is clear and used as antigen in various dilutions.

The following controls were tested: liver extract made in the same way from the liver of a case of miliary tuberculosis; liver from a case of tuberculous meningitis without tubercles in its substance; and the Wassermann antigen, alcoholic extract of heart. All gave negative results with positive reacting B.B.F. sera.

TESTS.-Children tested $v$. B.B.F. antigen:

Normal: 51 tested, 49 negative.

Rheumatic: 69 tested, 61 positive.

Others: 66 tested, 54 negative.

Adults.-Rheumatic cases: 69 tested, 54 positive. 
Researches on the Streptococci-Cech: Prague Cas. lek. ces., 1937, p. 583.-Experiments to clarify the ætiology of rheumatism. These experiments have shown that it is possible to sensitise rabbits with streptococcus vaccine, the best results being by the intravenous route; this sensitisation lasts about three months. By reinjection at the same point (joint) a local sensitisation may be induced. The symptoms that follow revaccination with the strepto-vaccine are of the ordinary inflammatory exudative character and altogether different from those observed in rheumatism. Cech as a result of his work cannot adopt either the views of Klinge (Klin. Woch., 1930), who regards the malady as a pure reaction of hypersensitisation of the mesenchyme, or the opinion that the streptococcus is the agent of rheumatism and that allergy is the pathogenic mechanism.

Chini and Magrassi: Policlinico, 1935, vol. x., p. 565 (Italy).-These authors have tested the skin reaction and the agglutinating power of the serum in eighty patients against the streptococci, among them an important proportion of cases of rheumatism. The results obtained do not constitute a demonstration of the streptococcal nature of rheumatism, but are due to foci of streptococcal origin having no relation to the rheumatic process.

VACIRA: Bull. Inst. Sier. Milan, 1935, vol. viii., p. 737.-A study of the relation of the streptococcus to acute rheumatism, leading to the conclusion that the histological structure of the Aschoff nodes is essentially different from the structure of the focal infiltrations that are met with in rabbits experimentally infected with streptococci. The latter correspond, on the contrary, with the nodes of Graeff in man infected with streptococci with localisation in the joints. The author has not found that streptococcal strains from rheumatic patients are more particularly arthrophilic than others. The arthrophilia of the strains of streptococci is solely related to their individual degree of virulence, and does not increase with repeated passage through joints. Among animals rendered allergic by vaccination or by chronic infection, manifestations with any relation to those of rheumatism in man are never seen. The conclusion is opposed to the hypothesis that the streptococcus is the causal agent of acute rheumatic polyarthritis in man.

Virus Infection-Andrei and Ravenna: Bull. Inst. Sier. Milan, 1935, vol. viii., p. 713.-These authors in experiments on 
480 rabbits have found that by injecting young animals intraperitoneally with the blood of patients with acute rheumatism there follows after an incubation of six weeks a thrombo-endocarditis that is transmissible in series from rabbit to rabbit up to five passages. They have never isolated any germ from the cardiac lesions or the blood, and conclude that the endocarditis is transmissible by contagion from one rabbit to another and that it is due to a filterable virus. This affection has never been seen in the normal control rabbits, but it has been found in rabbits injected with blood from normal subjects and those suffering from diseases other than rheumatism. Further work is in progress.

M. H. G.

\section{CORRESPONDENCE}

DeAr Sir,

\section{The Editor of The Rheumatic Diseases.}

I am very grateful for the review of my book, "The Synovial Membrane and the Synovial Fluid," which appeared in your journal.

Deeply convinced as I am of the shortcomings of human endeavour, I sincerely appreciate criticism of my own work. Unfortunately, Dr. Bywaters made errors which cast undeserved doubt on the work of qualified investigators quoted in my book. On p. 64, line 5 from the bottom, Dr. Bywaters writes: "Miller is quoted as saying that normal human joints are under a negative pressure of 6 to $12 \mathrm{~mm}$. of water. This would need a magician to prove; and unfortunately Miller is not listed in the bibliography." In my book, p. 92, line 7 from the bottom, the paragraph referred to reads as follows: "Miller has found that normal joints of living humans and dogs are under a negative pressure which amounts to about 6 to 12 centimetres of water." Dr. Bywaters mistook centimetres for millimetres. The method employed by the author quoted, far from being magical, is based on simple and generally accepted physical determination of differences in pressure. In my text, the printer's proof-reader substituted the English spelling of the name of the author as Miller. In the bibliography the name is spelled correctly: "Mueller, Walter: Biologie der Gelenke. Leipzig, Verlag von Johann Ambrosius Barth, Leipzig, 1929."

I would like to point out one other statement concerning the work of two authors besides my own which is misleading. On p. 63, line 12 from the bottom, we read, "He cites Curtis and Brunschwig that cases of osteo-arthritis show a high rate of absorption (borne out by his own case in Table III., which gave the highest phenolsulphonephthalein clearance). This is taken to mean an increased local blood supply." The authors quoted (p. 53) are not Curtis and Brunschwig, but Curtis and Brandenburg. Neither they nor myself interpreted the rate of absorption of phenolsulphonephthalein from the joints as an indication of increased blood supply. It is only pointed out that adhesions which destroy the synovial membrane decrease the rate of excretion.

You will understand that in requesting a correction of these misstatements I only act out of a feeling of obligation towards the authors whom I have quoted.

Thanking you for any courtesy, 